-Review-

\title{
Treatment and Prevention of Diabetic Ketoacidosis in Children and Adolescents
}

\author{
Yukashi Ohki \\ Department of Pediatrics, Nippon Medical School
}

\section{Introduction}

Ketoacidotic coma remains an important cause of mortality in children and adolescents with diabetes mellitus ${ }^{1}$. Despite this, it is preventable by educating patients and their attendants, except at first onset of the disease ${ }^{2}$. The management of ketoacidotic coma is important not only in patients with type 1 diabetes, but also with type 2 diabetes (so-called 'soft drink ketosis') $)^{3,4}$.

This review discusses aspects of the causes, the pathophysiology, the clinical features, the treatment and the prevention of diabetic ketoacidosis (DKA).

\section{Causes}

Precipitation factors leading to DKA are newly presented type 1 diabetes with $\beta$-cell depletion, so-called abrupt onset type 1 diabetes, omitted or decreased doses of insulin, and sick day (a febrile illness, acute stress from trauma or severe psychological stress that increase counter-regulatory hormone) (Table 1). Since urine glucose mass screening has been conducted in schools in Japan, the number of abrupt onset patients has decreased, which has contributed to the decrease in the number of deaths from DKA at onset in Japan ${ }^{5}$.

\section{Pathophysiology}

Kitabchi et al. ${ }^{6}$ described the pathogenesis of DKA
Table 1 Causes of DKA
1) Newly presented type 1 diabetes with $\beta$-cell depletion (abrupt onset)

2) Incorrect insulin dosage (omitted or decreased)

3) Sick day

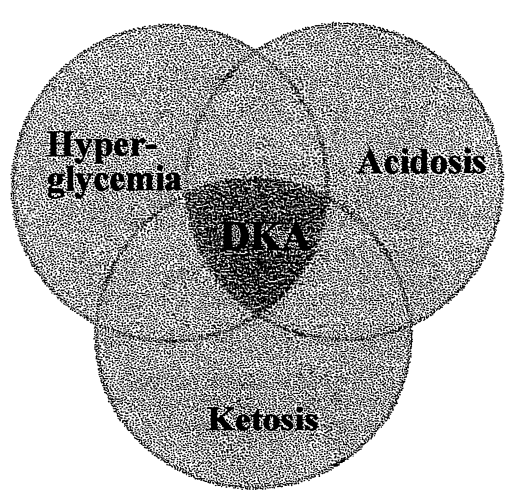

Fig. 1 Pathophysiology (1) - Diagnostic triad

simply as a diagnostic triad (Fig. 1). DKA is a metabolic derangement consisting of 3 concurrent abnormalities, which are high blood glucose, high levels of ketone bodies, and metabolic acidosis.

DKA is characterized by severe alterations in carbohydrate, lipid, and protein metabolism, mainly as a result of a lack of, or ineffectiveness of insulin, with concomitant elevations of counter-regulatory hormones (such as growth hormone, glucagon, glucocorticoid, catecholamines) due to stress (Fig. 2).

\section{1) Carbohydrate pathway}

First, in the carbohydrate pathway decreased insulin leads to increased glucose production and

Correspondence to Yukashi Ohki, Department of Pediatrics, Nippon Medical School, 1-1-5 Sendagi, Bunkyo-ku,

Tokyo 113-8603, Japan

E-mail: yukashi@nms.ac.jp

Journal Website (http://www.nms.ac.jp/jnms/) 


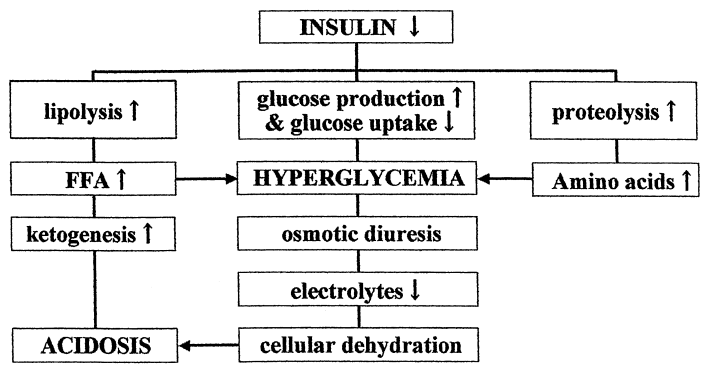

Fig. 2 Pathophysiology (2) - Carbohydrate, lipid and protein pathway

Table 2 Clinical features of DKA

1) Symptoms

polyuria, thirst, polydipsia, weight loss, abdominal pain, weakness, vomiting, air hunger, visual diaturbance, confusion

2) Signs

lethargy, dehydration, Kussmaul respiration, smell of ketones on breath, disordered consciousness or unconscious

decreased glucose uptake that results in hyperglycemia. Hyperglycemia then causes osmotic diuresis, loss of electrolytes and cellular dehydration.

\section{2) lipid pathway}

Second, in the lipid pathway increased lipolysis leads to increased free fatty acid, which stimulates gluconeogenesis and ketogenesis. These ketoacids are buffered by extracellular and cellular buffers, and generate endogenous bicarbonate, resulting in metabolic acidosis.

\section{3) protein pathway}

Third, in the protein pathway increased proteolysis leads to increased amino acids, which serve as major substrates for gluconeogenesis.

\section{Clinical features}

Based on this pathophysiology, the clinical features of DKA are shown in Table 2. The symptoms are: polyuria, thirst, polydipsia, weight loss, abdominal pain, weakness, vomiting, air hunger, visual disturbance and confusion. The signs are: lethargy, dehydration, Kussmaul respiration, smell of ketones on breath and disordered consciousness or unconsciousness ${ }^{7}$.
Table 3 Treatment of DKA (1) - Therapeutic goals

1) Improve circulatory volume and tissue perfusion

2) Correct electrolyte imbalances

3) Decrease serum glucose

4) Clear the serum and urine of ketoacids at a steady rate

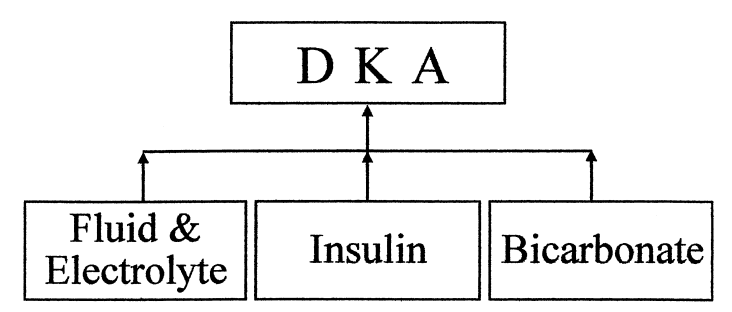

Fig. 3 Treatment of DKA (2) - Three kinds of replenishment

\section{Treatment}

The therapeutic goals of the treatment of DKA are: to improve circulatory volume and tissue perfusion, to correct electrolyte imbalances, to decrease serum glucose, and to clear the serum and urine of ketoacids at a steady rate (Table $\mathbf{3}$ ). Therefore, the treatment of DKA should involve 3 kinds of replenishment, which are fluid and electrolyte, insulin, and bicarbonate (Fig. 3). Vital signs and laboratory evaluation should be checked hourly.

\section{1) Fluid and electrolyte management (Table 4)}

The fluid replacement volume is the sum of half the losses and the maintenance volume. It is important to check any acute weight loss of the patient for the assumption the degree of dehydration, which ranges from 5 to $20 \%$, usually $10 \%$ in shocked patients. The maintenance dose is $60 \mathrm{ml} / \mathrm{kg} / 24$ hours. So, when a patient weighs $20 \mathrm{~kg}$ and has $10 \%$ acute weight loss, his replacement fluid volume for 24 hours will be $2,200 \mathrm{ml}$, which is 1,000 for half the losses plus 1,200 for the maintenance.

In the first 1 to 3 hours, normal saline should be given for immediate restitution of blood volume. This effectively removes the patient from the immediate consequences of potential or overt shock. 
Table 4 Fluid and electrolyte management

1) Volume
Half the fluid losses (\% of acute weight loss)
+ maintenance $(60 \mathrm{~m} l / \mathrm{kg} / 24$ hours)
2) Composition
a) First: normal $(0.9 \%)$ saline for $1-3$ hours
b) Next: half-normal $(0.45 \%)$ saline
c) After first urination: add $30 \mathrm{mEq} / l$ of $\mathrm{K}$
d) When BG falls below $250 \mathrm{mg} / \mathrm{dl}$ : add $5 \%$
dextrose
3) Infusion rate
a) First 8 hours: Half of the 24 hour volume
b) Next 16 hours: The other half

Treatment with hypotonic fluids and overload initially increases the extracellular $\mathrm{PH}$ more rapidly than intracellular $\mathrm{PH}$, activating the sodium proton pump and transporting sodium and water into the cell, leading to risks of cerebral edema ${ }^{8.9}$. Approximately $0.4 \sim 1 \%$ of children and adolescents with DKA develop cerebral edema with a high mortality/mobidity ${ }^{10}$.

Afterwards, normal saline is changed to half-normal saline with $30 \mathrm{mEq} / l$ of potassium on confirmation of the patient's first urination. Despite a total body potassium deficit, most patients with DKA have a serum potassium level at or above the upper limits of normal. These high levels occur because of a shift of potassium from the intracellular to the extracellular space due to acidemia, insulin deficiency, and hypertonicity ${ }^{11}$. Half of the potassium should be given as potassium chloride (15 meq/liter $\mathrm{KCL})$ and the other half as potassium phosphate (15 meq/liter K2HPO4). If there is any doubt about renal function, an electrocardiogram should be checked for peaked $\mathrm{T}$-waves before potassium is given.

When blood glucose level falls approximately 250 $\mathrm{mg} / \mathrm{d} l$ by insulin, $5 \%$ dextrose should be added, since both insulin and glucose are required to reverse glycogenolysis and ketogenesis.

Regarding the infusion rate, a half dose of 24 hours should be infused in the first 8 hours and another half in the next 16 hours.

\section{2) Insulin therapy (Table 5)}

Generally, blood glucose falls by approximately 10
Table 5 Insulin therapy
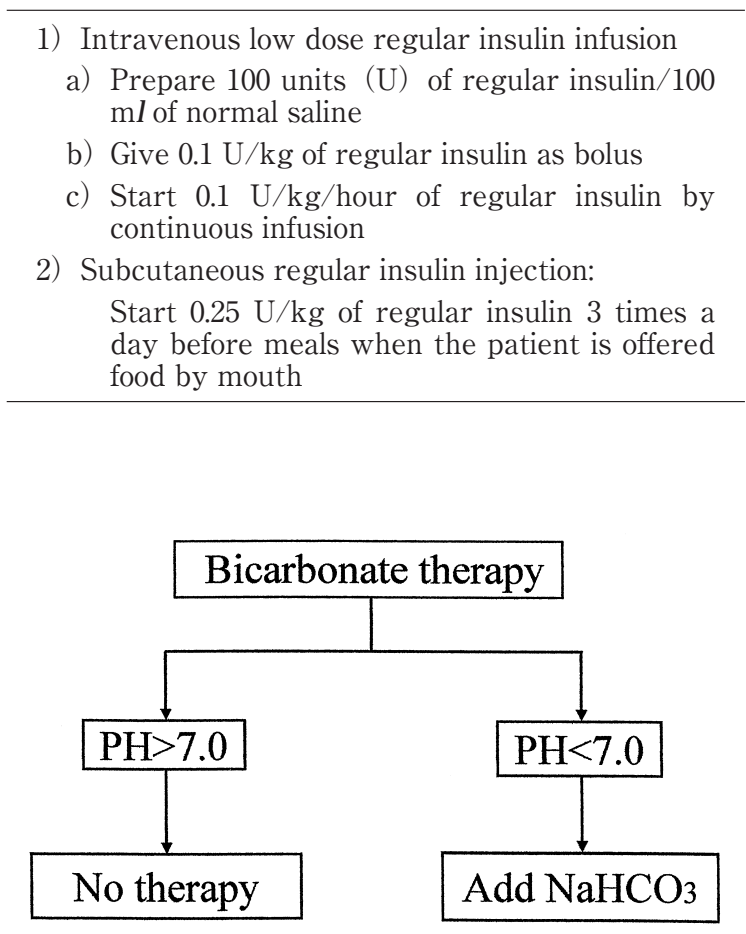

Fig. 4 Bicarbonate therapy

$\%$ per hour once the initial "dehydrated" blood glucose value is corrected with rehydration. So, intravenous low dose regular insulin infusion has become the standard method of treating $\mathrm{DKA}^{12}$.

The protocol is: prepare 100 units of regular insulin/ $100 \mathrm{~m} l$ of normal saline, give 0.1 units $/ \mathrm{kg}$ of regular insulin intravenously as bolus, and start 0.1 units/ $\mathrm{kg} /$ hour of regular insulin intravenously by continuous infusion. Subcutaneous regular insulin injection is started before meals, 3 times a day at a dose of 0.25 units $/ \mathrm{kg}$, when the patient is offered food by mouth.

When patients start complaining that they are hungry, it is usually prudent to begin refeeding. This is usually after the blood glucose falls to less than $250 \mathrm{mg} / \mathrm{d} l$.

\section{3) Bicarbonate therapy (Fig. 4)}

Since acetoacetate and betahydroxybutyrate are metabolizable anions, restoration of serum bicarbonate concentration will follow insulin administration in the absence of treatment with alkali containing solutions (Ketone bodies are converted to bicarbonate). Treatment with sodium 
bicarbonate should be restricted to patients with severe metabolic acidosis as indicated by an arterial $\mathrm{PH}$ of less than 7.0. When sodium bicarbonate is used, a $7 \%$ solution should be given at $1 \mathrm{ml} / \mathrm{kg}$ by slow intravenous infusion over several hours ${ }^{13}$. Potential dangers of bicarbonate include acute

平成 10 年 2 月: 血糖値 $(\mathrm{mg} / \mathrm{de})$

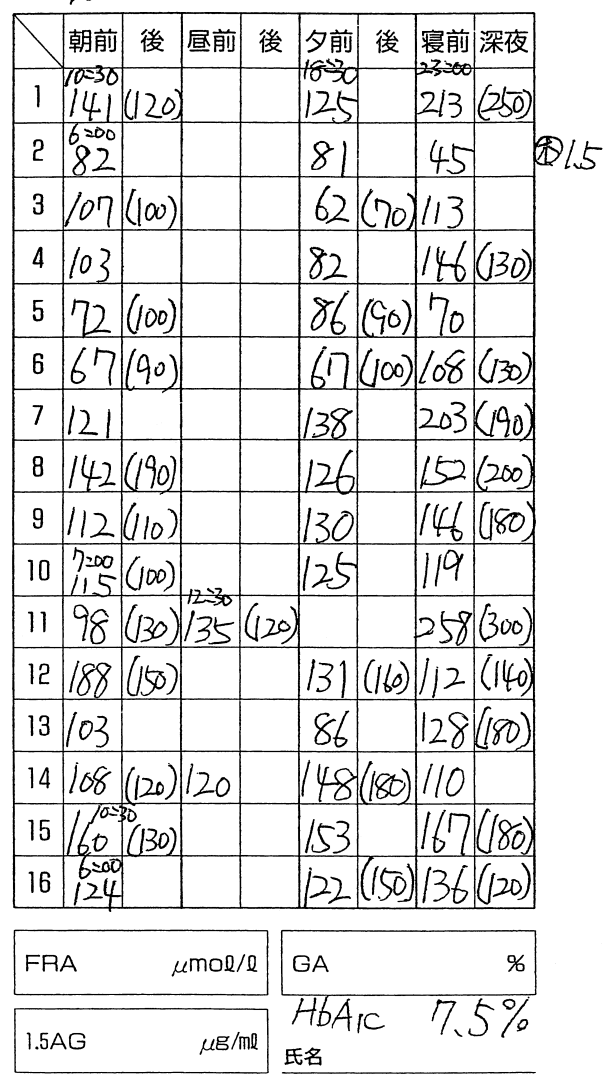

Fig. 5 SMBG note exacerbation of hypokalemia, tissue hypoxia due to reduced dissociation of oxyhemoglobin, sodium overload and increasing CSF acidosis.

\section{Prevention}

So, how do we prevent DKA, especially recurrent DKA?

\section{1) Self-monitoring blood glucose (SMBG)}

First, I emphasize the importance of forming a habit of self-monitoring blood glucose (SMBG) at least 2 times a day, before breakfast and before dinner. If possible, I also recommend SMBG before lunch and before sleep, a total of 4 times a day. And once a month, SMBG should be done 7 times a day, including the above-mentioned times plus 2 hours after each meal.

\section{2) Predicting blood glucose levels before SMBG}

Second, before SMBG, patients should practice predicting their blood glucose level from their physical condition. The difference between predicted

Table 6 Symptoms of mild/moderate hyperglycemia

1) A heavy feeling, a hot feeling, malaise

2) Thirst, polydipsia, polyuria

3) Headache, nausea, abdominal pain

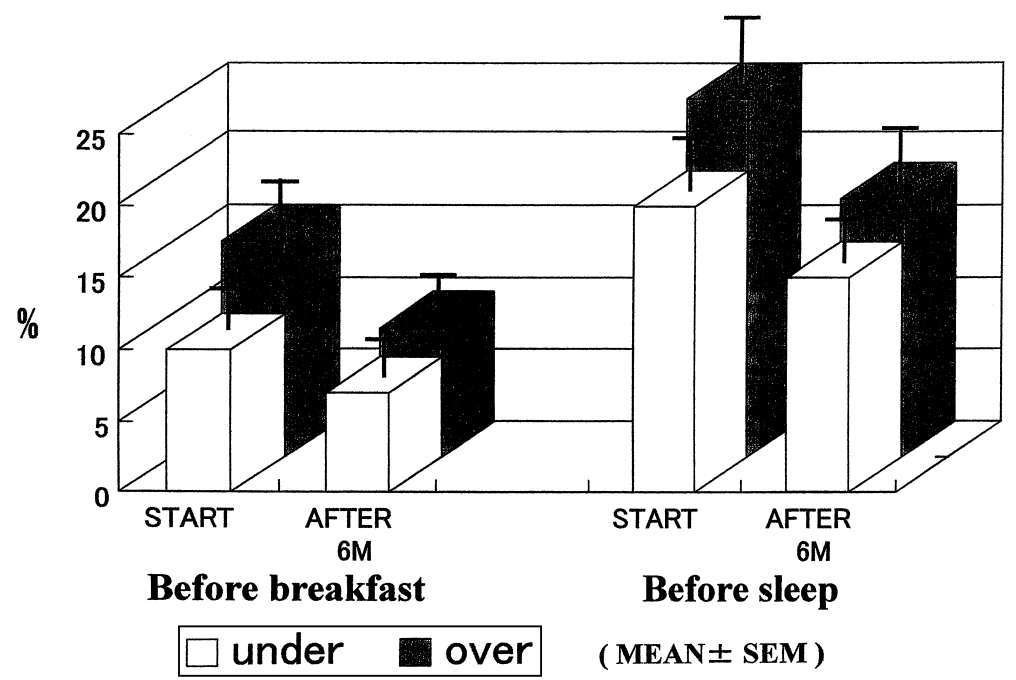

Fig. 6 Effect of 6 months' training in blood glucose prediction 
and measured values will decrease with practice.

Fig 5 shows the SMBG note of a 13-year-old boy whose HbAlc is $7.5 \%$. These Japanese 'Kanji' mean before breakfast, lunch, dinner and before sleep from left to right. He does SMBG 3 times a day-before breakfast, dinner and sleep. The numbers on the left are the actual measured values of blood glucose and those on the right in parentheses were predicted by the patient before SMBG. The 2 values are sometimes very close and sometimes differ. So, how do patients predict their blood glucose levels? The symptoms of hypoglycemia are well known, but those of mild to moderate hyperglycemia in which blood glucose levels are between 250 to $500 \mathrm{mg} / \mathrm{d} l$ depend on the person.

The most common symptoms of mild to moderate hyperglycemia are feeling heavy, feeling hot and malaise. Next are thirst, polydipsia, and polyuria. The patient sometimes has headache, nausea, and abdominal pain (Table 6). These symptoms are very important for prediction of hyperglycemia.

The mean difference between the actual blood glucose values and predicted values in 7 patients who kept records for 6 months before breakfast and before sleep is shown in Fig. 6. The records were divided into 2 groups, underestimated values and overestimated values, because if these groups were mixed, the data would offset each other and the error would become smaller. White bars are underestimated percentage to measured blood glucose values, and black bars are overestimated percentage. For instance, for the first 1 month the extent of underestimation was $10 \%$, which means when measured blood glucose was $100 \mathrm{mg} / \mathrm{d} l$, the predicted one was 90 .

It is effective for correcting predicted values if the patient knows the tends and characteristics of the previous prediction. You can see in Fig. 6 that the differences decreased after 6 months for both underand overestimated predicted values, and both before breakfast and before sleep.

Thus, it is very effective for patients to practice predicting their blood glucose level before SMBG based on their physical condition. The difference between predicted and measured values will decrease with more practice and this might be effective for preventing DKA.

I presented part this review at a lecture in a symposium at the $22^{\text {nd }}$ International Congress of Pediatrics (Amsterdam, 1998).

\section{References}

1. Basu A, Close CF, Jenkins D, et al:Persisting mortality in diabetic ketoacidosis. Diabet Med 1992; 10: 282-289.

2. Singh RK, Perros P, Frier BM: Hospital management of diabetic ketoacidosis. Are clinical guidelines implemented effectively? Diabetic Med 1997; 14: 482486.

3. Yamada K, Nonaka K: Diabetic ketoacidosis in young obese Japanese men. Diabetes Care 1996; 19: 671.

4. Westphal SA: The occurrence of diabetic ketoacidosis in non-insulin-dependent diabetes and newly diagnosed diabetic adults. Am J Med 1996; 101: 19-24.

5. Nishimura R, Matsushima M, Tajima N, et al:A major improvement in the prognosis of individuals with IDDM in the past 30 years in Japan. Diabetes Care 1996; 19: 758-760.

6. Kitabchi AE, Wall BM: Diabetic ketoacidosis. Med Clin North Am 1995; 79: 9-37.

7. Edge JA: Management of diabetic ketoacidosis in childhood. Bri J Hosp Med 1996; 55: 508-512.

8. Finberg L: Why do patients with diabetic ketoacidosis have cerebral swelling and why does treatment sometimes make it worse? Arch Pediatr Adol Med 1996; 150: 785-786.

9. Harris GD, Fiordalisi I, Yu C:Maintaining normal intracranial pressure in a rabbit model during treatment of severe diabetic ketoacidemia. Life Sciences 1996; 59: 1695-1702.

10. Diabetic ketoacidosis. "International society for pediatric and adolescent diabetes (ISPAD) consensus guidelines for the management of type 1 diabetes mellitus in children and adolescents" (Swift PGF, ed) 2000; pp63-73, Medical Forum International, Zeist, Netherlands.

11. Umpierrez GE, Beth M, Kitabuchi AE: Diabetic ketoacidosis and hyperglycemic hyperosmolar syndrome. Diabetes Spectrum 2002; 15: 28-36.

12. Kitabchi AE, Ayyagari V, Guerra SMO, et al: The efficacy of low dose versus conventional therapy of insulin for treatment if diabetic ketoacidosis. Ann Int med 1976; 84: 633-638.

13. Brink SJ: Presentation and ketoacidosis. "Childhood and adolescent diabetes" (Kelnar CJH, ed) 1995; pp226-240, Chapman and Hall Medical, London.

(Received, December 13, 2002)

(Accepted, February 7, 2003) 